\title{
Reciprocal link for a three-component Camassa-Holm type equation
}

Nianhua Li

To cite this article: Nianhua Li (2016) Reciprocal link for a three-component CamassaHolm type equation, Journal of Nonlinear Mathematical Physics 23:2, 150-156, DOI: https://doi.org/10.1080/14029251.2016.1161257

To link to this article: https://doi.org/10.1080/14029251.2016.1161257

Published online: 04 January 2021 


\title{
Reciprocal link for a three-component Camassa-Holm type equation
}

\author{
Nianhua Li \\ School of Mathematics, Huaqiao University \\ Quanzhou, Fujian 362021, People's Republic of China \\ linianh@hqu.edu.cn
}

Received 3 September 2015

Accepted 4 January 2016

\begin{abstract}
A reciprocal transformation is introduced for a three-component Camassa-Holm type equation and it is showed that the transformed system is a reduction of the first negative flow in a generalized MKdV hierarchy.

Keywords: Camassa-Holm type equation; Reciprocal transformation; Hamiltonian structure
\end{abstract}

2000 Mathematics Subject Classification: 37K10, 35Q51, 35Q58

\section{Introduction}

The Camassa-Holm $(\mathrm{CH})$ equation

$$
u_{t}-u_{x x t}+3 u u_{x}+2 k u_{x}=2 u_{x} u_{x x}+u u_{x x x}
$$

was derived by Camassa and Holm as a model for unidirectional dispersive shallow-water motion using an asymptotic expansion directly in the Hamiltonian for Euler's equations [1,2]. It is completely integrable with a Lax pair, bi-Hamiltonian structure and infinitely many conserved quantities. The equation can be solved by the inverse scattering transformation [4], and is found to admit multi-soliton solutions and algebro-geometric solutions [10,21,23]. In particular, the discovery of peakons [1,2] (if $k=0$ ) makes the $\mathrm{CH}$ equation a subject of extensive research in recent years. The peakons are weak solutions in certain Sobolov space and of interest for water wave theory as well as from the point of view of general analysis for PDEs. Besides, the $\mathrm{CH}$ equation is linked to the first negative flow in the KdV hierarchy $[7,17]$ via a reciprocal transformation. It is remarked that reciprocal transformations, originated from the study of invariance theory in gasdynamics and magneto-gasdynamics, have diverse physical applications and been used extensively to reveal connections between integrable hierarchies (see e.g., $[16,26]$ and references therein).

Subsequently, various other $\mathrm{CH}$ type equations (possessing peakon solutions) are proposed and studied, to mention just a few we cite the Degasperis-Procesi (DP) equation, the Novikov equation and the Geng-Xue equation [5, 6, 9, 14, 15, 20,24]. While those systems are completely integrable, they have some nonstandard features such as the weak Painlevé property [5,11,13]. Reciprocal transformations are a useful tool to connect the hierarchies displaying weak Painlevé behaviour with the hierarchies displaying the standard (strong) Painlevé test of Weiss, Tabor and Carnevale (WTC) [12]. Just as the $\mathrm{CH}$ equation is reciprocally linked to the negative $\mathrm{KdV}$ equation, the DP equation, the Novikov equation and the Geng-Xue equation are reciprocally connected to the negative flows in the Kaup-Kupershmidt hierarchy, the Sawada-Kotera hierarchy and the modified Boussinesq hierarchy respectively $[15,20]$. 
Recently, a three-component $\mathrm{CH}$ type system admitting the linear problem

$$
\psi_{x}=\left(\begin{array}{ccc}
0 & 1 & 0 \\
1+\lambda v & 0 & u \\
\lambda w & 0 & 0
\end{array}\right) \psi
$$

and $N$-peakon solutions was proposed by Geng and Xue [8], that is

$$
\begin{aligned}
& u_{t}=-v p_{x}+u_{x} q+\frac{3}{2} u q_{x}-\frac{3}{2} u\left(p_{x} r_{x}-p r\right), \\
& v_{t}=2 v q_{x}+v_{x} q, \\
& w_{t}=v r_{x}+w_{x} q+\frac{3}{2} w q_{x}+\frac{3}{2} w\left(p_{x} r_{x}-p r\right),
\end{aligned}
$$

where

$$
\begin{aligned}
u & =p-p_{x x} \\
v & =\frac{1}{2}\left(q_{x x}-4 q+p_{x x} r_{x}-r_{x x} p_{x}+3 p_{x} r-3 p r_{x}\right), \\
w & =r_{x x}-r .
\end{aligned}
$$

The bi-Hamiltonian structure as well as infinitely many conserved quantities and the dynamical system for the $N$-peakon solutions of the system are obtained $[8,18]$.

Very recently, based on the following spectral problem

$$
\phi_{x}=\left(\begin{array}{ccc}
0 & 0 & 1 \\
\lambda m_{1} & 0 & \lambda m_{3} \\
1 & \lambda m_{2} & 0
\end{array}\right) \phi,
$$

Liu, Popowicz and the present author constructed another three-component $\mathrm{CH}$ type system [19]

$$
\begin{aligned}
& m_{1 t}+u_{2} g m_{1 x}-m_{3}\left(u_{2 x} f-u_{2} g\right)-m_{1}\left(3 u_{2} f-m_{3} u_{2}\right)=0, \\
& m_{2 t}+u_{2} g m_{2 x}+m_{2}\left(3 u_{2 x} g+m_{3} u_{2}\right)=0, \\
& m_{3 t}+u_{2} g m_{3 x}-m_{3}\left(2 u_{2} f+u_{2 x} g-m_{3} u_{2}\right)=0, \\
& m_{i}=u_{i}-u_{i x x}, i=1,2,3, \quad f=u_{3}-u_{1 x}, \quad g=u_{1}-u_{3 x} .
\end{aligned}
$$

We showed that the $\mathrm{CH}$ type system (1.6) admits a bi-Hamiltonian structure as well as infinitely many conserved quantities. It was pointed out that the spectral problem (1.5) may be transformed to the spectral problem (1.2) by a gauge transformation [19], and we will only consider to construct a reciprocal transformation for the system (1.6).

The purpose of this paper is to construct a reciprocal transformation for the three-component $\mathrm{CH}$ type equation (1.6), and establish a relationship between the transformed system and a generalized MKdV hierarchy. These will be done in Sec. 2 and Sec. 3.

\section{A reciprocal transformation}

In this section, we will construct a reciprocal transformation for the $\mathrm{CH}$ type system (1.6). As pointed out in [19], the three-component system (1.6) is exactly the compatibility condition of the 
spectral problem (1.5) and associated auxiliary problem

$$
\phi_{t}=\left(\begin{array}{ccc}
-u_{2} f & \lambda^{-1} u_{2} & -u_{2} g \\
\lambda^{-1} f-\lambda m_{1} u_{2} g & u_{2} f+u_{2 x} g-\lambda^{-2} & \lambda^{-1} g-\lambda m_{3} u_{2} g \\
-u_{2 x} f & \lambda^{-1} u_{2 x}-\lambda m_{2} u_{2} g & -u_{2 x} g
\end{array}\right) \phi .
$$

Based on the above Lax pair, an infinite sequence of conservation laws may be constructed. In particular, we have

$$
\left(\left(m_{2} m_{3}\right)^{\frac{1}{2}}\right)_{t}=-\left(\left(m_{2} m_{3}\right)^{\frac{1}{2}} u_{2} g\right)_{x}
$$

which introduces a reciprocal transformation

$$
d y=a d x-a u_{2} g d t, \quad d \tau=d t,
$$

where $a=\left(m_{2} m_{3}\right)^{\frac{1}{2}}$.

On the one hand, writing the column vector $\phi$ in components as $\phi=\left(\phi_{1}, \phi_{2}, \phi_{3}\right)^{T}$ and elimating $\phi_{3}$ from the spectral problem (1.5), we get

$$
\phi_{1 x x}-\phi_{1}=\lambda m_{2} \phi_{2}, \quad \phi_{2 x}=\lambda m_{1} \phi_{1}+\lambda m_{3} \phi_{1 x}
$$

which in the new variable can be written as

$$
\begin{aligned}
& \phi_{1 y y}+\frac{a_{y}}{a} \phi_{1 y}-\frac{1}{a^{2}} \phi_{1}=\lambda \frac{1}{m_{3}} \phi_{2}, \\
& \phi_{2 y}=\lambda \frac{m_{1}}{a} \phi_{1}+\lambda m_{3} \phi_{1 y} .
\end{aligned}
$$

Setting $b=e^{-\partial_{y}^{-1} \frac{m_{1}}{a m_{3}}}$ and after a gauge transformation

$$
\phi_{1}=b \varphi_{1}, \quad \phi_{2}=m_{3} b \varphi_{2},
$$

the spectral problem (2.3)-(2.4) is transformed to

$$
\begin{aligned}
& \varphi_{1 y y}-Q_{2} \varphi_{1 y}-Q_{1} \varphi_{1}=\lambda \varphi_{2}, \\
& \varphi_{2 y}=\lambda \varphi_{1 y}+Q_{3} \varphi_{2},
\end{aligned}
$$

where

$$
Q_{1}=-\frac{b_{y y}}{b}-\frac{a_{y} b_{y}}{a b}+\frac{1}{a^{2}}, \quad Q_{2}=-2 \frac{b_{y}}{b}-\frac{a_{y}}{a}, \quad Q_{3}=\frac{m_{1}}{a m_{3}}-\frac{m_{3 y}}{m_{3}} .
$$

Eliminating $\varphi_{2}$ between (2.5) and (2.6), we find

$$
\left(\partial_{y}^{2}+u \partial_{y}+v+\partial_{y}^{-1} w\right) \varphi_{1}=\lambda^{2} \varphi_{1} .
$$

The hierarchy associated with this spectral problem is an extended MKdV hierarchy which is related to an extended KdV hierarchy (the Yajima-Oikawa hierarchy $[3,27]$ ) via a Miura transformation 
[22, 25]. In fact the relationship between $\left(Q_{1}, Q_{2}, Q_{3}\right)$ and $(u, v, w)$ is established by the following Miura transformation

$$
u=-\left(Q_{2}+Q_{3}\right), \quad v=Q_{2} Q_{3}-Q_{1}+Q_{3 y}, \quad w=Q_{1} Q_{3}-\left(Q_{2} Q_{3}\right)_{y}-Q_{3 y y}
$$

which may be obtained from factorizing the Lax operator in (2.8), that is

$$
\partial_{y}^{2}+u \partial_{y}+v+\partial_{y}^{-1} w=\partial_{y}^{-1}\left(\partial_{y}-Q_{3}\right)\left(\partial_{y}^{2}-Q_{2} \partial_{y}-Q_{1}\right) .
$$

Furthermore, the spectral problem (2.5)-(2.6) may be reduced to the spectral problem of the MKdV hierarchy as $Q_{1}=Q_{2}=0$, thus we call the hierarchy associated with the spectral problem (2.5)-(2.6) a generalized MKdV hierarchy.

Similarly, the auxiliary problem (2.1) may be changed to the following form

$$
\begin{aligned}
& \varphi_{1 \tau}=\lambda^{-1} q_{3} \varphi_{2}, \\
& \varphi_{2 \tau}=\left(-\lambda^{-2}+q_{3}\right) \varphi_{2}+\lambda^{-1} q_{2} \varphi_{1 y}+\lambda^{-1} q_{1} \varphi_{1},
\end{aligned}
$$

where

$$
q_{1}=\frac{u_{3}-u_{1 y} a}{m_{3}}-\frac{m_{1}}{m_{3}^{2}}\left(u_{1}-u_{3 y} a\right), \quad q_{2}=\frac{a}{m_{3}}\left(u_{1}-u_{3 y} a\right), \quad q_{3}=m_{3} u_{2} .
$$

Then the Lax pair (1.5)-(2.1) for the three-component $\mathrm{CH}$ type system (1.6) is transformed to

$$
\varphi_{y}=\left(\begin{array}{ccc}
0 & 1 & 0 \\
Q_{1} & Q_{2} & \lambda \\
0 & \lambda & Q_{3}
\end{array}\right) \varphi
$$

and

$$
\varphi_{\tau}=\left(\begin{array}{ccc}
0 & 0 & \lambda^{-1} q_{3} \\
0 & q_{3} & \lambda^{-1}\left(q_{3 y}+Q_{3} q_{3}\right) \\
\lambda^{-1} q_{1} & \lambda^{-1} q_{2} & -\lambda^{-2}+q_{3}
\end{array}\right) \varphi
$$

On the other hand, under the transformation (2.2), the $\mathrm{CH}$ type system (1.6) is transformed to

$$
\begin{array}{ll}
m_{1 \tau}=m_{1}\left(3 u_{2} f-m_{3} u_{2}\right)+m_{3}\left(u_{2 y} a f-u_{2} g\right), & m_{1}=u_{1}-a\left(u_{1 y} a\right)_{y}, \\
m_{2 \tau}=-m_{2}\left(3 u_{2 y} a g+m_{3} u_{2}\right), & m_{2}=u_{2}-a\left(u_{2 y} a\right)_{y}, \\
m_{3 \tau}=m_{3}\left(2 u_{2} f+u_{2 y} a g-m_{3} u_{2}\right), & m_{3}=u_{3}-a\left(u_{3 y} a\right)_{y},
\end{array}
$$

where

$$
f=u_{3}-a u_{1 y}, \quad g=u_{1}-a u_{3 y} .
$$

With the help of the Liouville transformation

$$
\left(\begin{array}{l}
Q_{1} \\
Q_{2} \\
Q_{3}
\end{array}\right)=\left(\begin{array}{c}
\left(m_{2} m_{3}\right)^{-1}\left[\left(\frac{m_{1}}{m_{3}}\right)_{x}+1-\left(\frac{m_{1}}{m_{3}}\right)^{2}\right] \\
\left(m_{2} m_{3}\right)^{-\frac{3}{2}}\left[2 m_{1} m_{2}-\frac{1}{2}\left(m_{2} m_{3}\right)_{x}\right] \\
m_{2}^{-\frac{1}{2}} m_{3}^{-\frac{3}{2}}\left(m_{1}-m_{3 x}\right)
\end{array}\right), \quad y=\int_{-\infty}^{x}\left(m_{2} m_{3}\right)^{\frac{1}{2}} d x
$$


and through tedious but direct calculations, we obtain the system for $Q_{1}, Q_{2}, Q_{3}$ as follows

$$
\begin{aligned}
& Q_{1 \tau}=Q_{1} q_{3}-q_{1}, \quad S_{1}=-1, \\
& Q_{2 \tau}=2 q_{3 y}+Q_{3} q_{3}-q_{2}, \quad S_{2}=0, \\
& Q_{3 \tau}=-Q_{3} q_{3}+q_{2}, \quad S_{3}=-1 \text {, }
\end{aligned}
$$

where

$$
\begin{aligned}
& S_{1}=\left[\left(\partial_{y}+Q_{3}-Q_{2}\right)\left(\partial_{y}+Q_{3}\right)-Q_{1}\right] q_{3}, \\
& S_{2}=\left(-\partial_{y}+Q_{3}\right) q_{1}-Q_{1} q_{2}, \\
& S_{3}=\left(-\partial_{y}+Q_{3}-Q_{2}\right) q_{2}-q_{1} .
\end{aligned}
$$

Direct calculation shows that the compatibility condition of the Lax pair (2.11)-(2.12) yields just the transformed system (2.13). Therefore we conclude that, under the reciprocal transformation (2.2), the three-component $\mathrm{CH}$ type system (1.6) with a Lax pair (1.5)-(2.1) are changed to (2.13) and its Lax pair (2.11)-(2.12), accordingly.

\section{The link between the transformed $\mathrm{CH}$ type system and the generalized MKdV hierarchy}

According to [25], the extended MKdV hierarchy associated with the spectral problem (2.8) may be formulated as a bi-Hamiltonian system admitting the Hamiltonian pair

$$
\begin{aligned}
& \mathscr{J}_{1}=\left(\begin{array}{ccc}
0 & 0 & 2 \partial_{y} \\
0 & 2 \partial_{y} & \partial_{y}^{2}+u \partial_{y} \\
2 \partial_{y}-\partial_{y}^{2}+\partial_{y} u & 0
\end{array}\right), \\
& \mathscr{J}_{2}=\left(\begin{array}{ccc}
6 \partial_{y} & * & * \\
4 u \partial_{y} & 2 \partial_{y}^{3}+2 u \partial_{y} u+\partial_{y} v+v \partial_{y} & * \\
2 \partial_{y}^{3}-2 \partial_{y} u \partial_{y}+2 v \partial_{y} & \theta_{1} & \theta_{2}
\end{array}\right) \text {, }
\end{aligned}
$$

where

$$
\begin{aligned}
& \theta_{1}=-\partial_{y}^{4}+\partial_{y}^{3} u+\partial_{y} u \partial_{y}^{2}-\partial_{y} u \partial_{y} u-v \partial_{y}^{2}+v \partial_{y} u+2 w \partial_{y}+\partial_{y} w, \\
& \theta_{2}=\partial_{y} u w+u w \partial_{y}+w \partial_{y}^{2}-\partial_{y}^{2} w
\end{aligned}
$$

and the omitted terms in the operator $\mathscr{J}_{2}$ are determined by the skew-symmetry. Taken account of the Miura transformation (2.9), the compatible Hamiltonian operators for the generalized MKdV hierarchy associated with the spectral problem (2.11) (equivalent to the spectral problem (2.5)-(2.6)) may be obtained, which are

$$
\tilde{J}_{i}=\mathscr{F}^{-1} \mathscr{J}_{i} \mathscr{F}^{-1 *}, i=1,2, \quad \mathscr{F}=\left(\begin{array}{ccc}
0 & -1 & -1 \\
-1 & Q_{3} & \partial_{y}+Q_{2} \\
Q_{3} & -\partial_{y} Q_{3} & Q_{1}-\partial_{y} Q_{2}-\partial_{y}^{2}
\end{array}\right) .
$$

Now we may construct the first negative flow in this generalized MKdV hierarchy, which reads as

$$
\left(\begin{array}{l}
Q_{1} \\
Q_{2} \\
Q_{3}
\end{array}\right)_{\tau}=\mathscr{F}^{-1} \mathscr{J}_{1}\left(\begin{array}{l}
A \\
B \\
C
\end{array}\right), \quad \mathscr{F}^{-1} \mathscr{J}_{2}\left(\begin{array}{l}
A \\
B \\
C
\end{array}\right)=0
$$


In order to establish the link between the transformed system (2.13) and the first negative flow (3.1) in the generalized MKdV hierarchy, we introduce

$$
\begin{aligned}
A & =\frac{1}{4}\left(Q_{2}+Q_{3}\right) \partial_{y}^{-1}\left(S_{1}-S_{3}\right)-\frac{1}{2} \partial_{y}^{-1} S_{2}+\frac{1}{4}\left(S_{1}+S_{3}\right)-Q_{3} q_{3 y}-Q_{3}^{2} q_{3}, \\
B & =\frac{1}{2} \partial_{y}^{-1}\left(S_{1}-S_{3}\right)-Q_{3} q_{3}, \\
C & =-q_{3},
\end{aligned}
$$

(here all integration constants are assumed to be zero). Then substituting (3.2) into (3.1), the first negative flow is reformulated as

$$
\left(\begin{array}{l}
Q_{1} \\
Q_{2} \\
Q_{3}
\end{array}\right)_{\tau}=\left(\begin{array}{c}
Q_{1} q_{3}-q_{1} \\
2 q_{3 y}+Q_{3} q_{3}-q_{2} \\
-Q_{3} q_{3}+q_{2}
\end{array}\right), \quad \mathscr{F}^{-1} \mathscr{K}\left(\begin{array}{c}
S_{1} \\
S_{2} \\
S_{3}
\end{array}\right)=0
$$

where

$$
\mathscr{K}=\left(\begin{array}{ccc}
\frac{1}{2} \partial_{y} u \partial_{y}^{-1}-\frac{1}{2} \partial_{y} & -3 & \frac{3}{2} \partial_{y}-\frac{1}{2} \partial_{y} u \partial_{y}^{-1} \\
Q_{3} \partial_{y}+v+\frac{1}{2} v_{y} \partial_{y}^{-1} & -2 u & u \partial_{y}-\partial_{y}^{2}-v-\frac{1}{2} v_{y} \partial_{y}^{-1} \\
\chi_{1} & \partial_{y} u-\partial_{y}^{2}-v & \chi_{2}
\end{array}\right)
$$

herein

$$
\begin{aligned}
& \chi_{1}=-Q_{3} \partial_{y}^{2}-\left(2 Q_{3 y}+Q_{2} Q_{3}\right) \partial_{y}+\frac{1}{2}\left(3 w+w_{y} \partial_{y}^{-1}\right), \\
& \chi_{2}=\partial_{y}^{3}+v \partial_{y}-\partial_{y} u \partial_{y}-\frac{1}{2}\left(3 w+w_{y} \partial_{y}^{-1}\right),
\end{aligned}
$$

with $(u, v, w)$ given by (2.9).

From the definitions of $A, B, C$, it is easy to see that $S_{1}=S_{3}=-1$ and $S_{2}=0$ imply $\mathscr{K}\left(S_{1}, S_{2}, S_{3}\right)^{T}=0$. Therefore the transformed system (2.13) may be regarded as a reduction of the first negative flow (3.3) in the generalized MKdV hierarchy.

\section{Acknowledgments}

We would like to thank the anonymous Referees for their helpful comments. This work is supported by the National Natural Science Foundation of China (Grant Nos. 11271366, 11331008 and 11505064) and the Initial Founding of Scientific Research for the introduction of talents of Huaqiao University (Project No. 14BS314).

\section{References}

[1] R. Camassa and D. D. Holm, An integrable shallow water equation with peaked solitons, Phys. Rev. Lett. 71 (1993) 1661-1664.

[2] R. Camassa, D. D. Holm and J. M. Hyman, A new integrable shallow water equation, Adv. Appl. Mech. 31 (1994) 1-33.

[3] Y. Cheng, Constraints of the Kadomtsev-Petviashvili hierarchy, J. Math. Phys. 33 (1992) 3774-3782.

[4] A. Constantin, V. S. Gerdjikov and R. I. Ivanov, Inverse Scattering transform for the Camassa-Holm equation, Inverse Problems 22 (2006) 2197-2207.

[5] A. Degasperis, D. D. Holm and A. N. W. Hone, A new integrable equation with peakon solutions, Theor. Math. Phys. 133 (2002) 1463-1474. 
[6] A. Degasperis and M. Procesi, Asymptotic integrability Symmetry and Perturbation Theory ed A. Degasperis and G. Gaeta (Singapore, World Scientific, 1999) pp. 23-37.

[7] B. Fuchssteiner, Some tricks from the symmetry-toolbox for nonlinear equations: generalizations of the Camassa-Holm equation, Phys. D 95 (1996) 229-243.

[8] X. G. Geng and B. Xue, A three-component generalization of Camassa-Holm equation with N-peakon solutions, Adv. Math. 226 (2011) 827-839.

[9] X. G. Geng and B. Xue, An extension of integrable peakon equations with cubic nonlinearity, Nonlinearity 22 (2009) 1847-1856.

[10] F. Gesztesy and H. Holden, Algebro-geometric solutions of the Camassa-Holm hierarchy, Rev. Mat. Iberoamericana 19 (2003) 73-142.

[11] C. Gilson and A. Pickering, Factorization and Painlevé analysis of a class of nonlinear third-order partial differential equations, J. Phys. A: Math. Gen. 28 (1995) 2871-2888.

[12] A. N. W. Hone, Reciprocal transformations, Painlevé property and solutions of energy-dependent Schrödinger hierarchies, Phys. Lett. A 249 (1998) 46-54.

[13] A. N. W. Hone, The associated Camassa-Holm equation and the KdV equation, J. Phys. A: Math. Gen. 32 (1999) L307-314.

[14] A. N. W. Hone, H. Lundmark and J. Szmigielski, Explicit multipeakon solutions of Novikov's cubically nonlinear integrable Camassa-Holm type equation, Dyn. PDE 6 (2009) 253-289.

[15] A. N. W. Hone and J. P. Wang, Prolongation algebras and Hamiltonian operators for peakon equations, Inverse Problems 19 (2003) 129-145.

[16] J. G. Kingston and C. Rogers, Reciprocal Bäcklund transformations of conservation laws, Phys. Lett. A 92 (1982) 261-264.

[17] J. Lenells, The correspondence between KdV and Camassa-Holm, IMRN (International Mathematical Research Notices) 71 (2004) 3797-3811.

[18] N. H. Li and Q. P. Liu, Bi-Hamiltonian structure of a three-component Camassa-Holm type equation, J. Nonlinear Math. Phys. 20 (2013) 126-134.

[19] N. H. Li, Q. P. Liu and Z. Popowicz, A four-component Camassa-Holm type hierarchy, J. Geom. Phys. 85 (2014) 29-39.

[20] N. H. Li and X. X. Niu, A reciprocal transformation for the Geng-Xue equation. J. Math. Phys. 55 (2014) 053505.

[21] Y. S. Li and J. E. Zhang, The multiple-soliton solution of the Camassa-Holm equation, Proc. R. Soc. Lond. A 460 (2004) 2617-2627.

[22] Q. P. Liu, Modifications of $k$-constrained KP hierarchy, Phys. Lett. A 187 (1994) 373-381.

[23] Y. Matsuno, Parametric representation for the multisoliton solution of the Camassa-Holm equation, J. Phys. Soc. Japan 74 (2005) 1983-1987.

[24] V. S. Novikov, Generalisations of the Camassa-Holm equation, J. Phys. A: Math. Theor. 42 (2009) 342002.

[25] W. Oevel and W. Strampp, Constrained KP hierarchy and bi-Hamiltonian structure, Commun. Math. Phys. 157 (1993) 51-81.

[26] C. Rogers, W. K. Schief and A. Szereszewski, Loop soliton interaction in an integrable nonlinear telegraphy model: reciprocal and Bäcklund transformations, J. Phys. A: Math. Theor. 43 (2010) 385210.

[27] N. Yajima and M. Oikawa, Formation and interaction of sonic-Langmuir solitons, Prog. Theor. Phys. 56 (1976) 1719-1739. 